\title{
Drug delivery system based on dendritic nanoparticles for enhancement of intravesical instillation
}

Xuefeng Qiu',2,*

Kai Cao ${ }^{1,2, *}$

Tingsheng $\operatorname{Lin}^{1,2}$

Wei Chen'

Ahu Yuan ${ }^{2}$

Jinhui $\mathrm{Wu}^{2}$

Yiqiao $\mathrm{Hu}^{2}$

Hongqian Guo'

'Department of Urology, Drum Tower Hospital, Medical School of Nanjing University, Institute of Urology, Nanjing University, Nanjing, China; ${ }^{2}$ State Key Laboratory of Pharmaceutical Biotechnology, Medical School of Nanjing University, Nanjing, China

*These authors contributed equally to this work
This article was published in the following Dove Press journal:

International Journal of Nanomedicine

10 October 2017

Number of times this article has been viewed

\begin{abstract}
Intravesical instillation of antitumor agents following transurethral resection of bladder tumors is the standard strategy for the treatment of superficial bladder cancers. However, the efficacy of current intravesical instillation is limited partly due to the poor permeability of the urothelium. We therefore aimed to develop a high-penetrating, target-releasing drug delivery system to improve the efficacy of intravesical instillation. PAMAM, a dendrimer, were conjugated with polyethylene glycol (PEG) to form PEG-PAMAM complex as a nanocarrier. Doxorubicin (DOX) was then encapsulated into PEG-PAMAM to generate DOX-loaded PEGPAMAM nanoparticles (PEG-PAMAM-DOX). Our results indicated that the PEG-PAMAM was a stable nanocarrier with small size and great biosafety. The release of DOX from PEGPAMAM-DOX was sluggish but could be effectively triggered in an acid microenvironment $(\mathrm{pH}=5.0)$. As a drug carrier, PEG-PAMAM could penetrate mice bladder urothelium effectively and increase the amount of DOX within the bladder wall after intravesical instillation. The antitumor effect of PEG-PAMAM-DOX was evaluated using an orthotopic bladder cancer model in mice. Compared to free DOX, PEG-PAMAM-DOX showed significantly improved efficacy of DOX for intravesical instillation with limited side effects. In conclusion, we successfully developed a PEG-PAMAM-based drug delivery system to enhance the antitumor effect of intravesical instillation.
\end{abstract}

Keywords: bladder cancer, intravesical instillation, chemotherapy, dendrimer, PAMAM, penetration, acid microenvironment, target-releasing

\section{Introduction}

Bladder cancer is one of the most common human malignancy worldwide. ${ }^{1}$ Superficial or nonmuscular-invasive bladder cancer (NMIBC) accounts for $>70 \%$ at initial diagnosis. ${ }^{2}$ The current standard treatment strategy for NMIBC is transurethral resection of bladder tumors (TURBT), followed by intravesical instillation of antitumor agents. ${ }^{3}$ However, recurrence rates in patients with NMIBC range from $31 \%$ to $78 \%$ within 5 years from the initial diagnosis in the low- and high-risk subgroups, respectively. ${ }^{4}$ Such high recurrence rates might be potentially attributed to the low efficacy of current intravesical instillation. ${ }^{5}$

The urothelium comprises basal cells, intermediate cells, and the luminal surface bladder permeability barrier (BPB), which is composed of umbrella cells joined by tight junctions, densely packed plaques, and the glycosaminoglycan mucin layer. ${ }^{5}$ It is the BPB that causes the low permeability of the urothelium to antitumor agents. Therefore, a great deal of efforts have been made to enhance the permeability of antitumor agents through the bladder wall. Physical approaches such as electromotive drug 
A

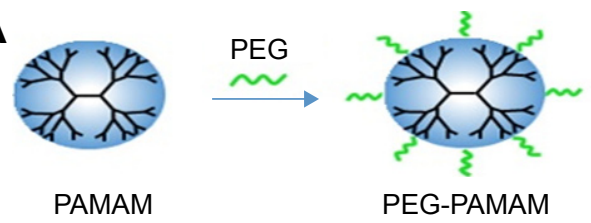

PAMAM

C
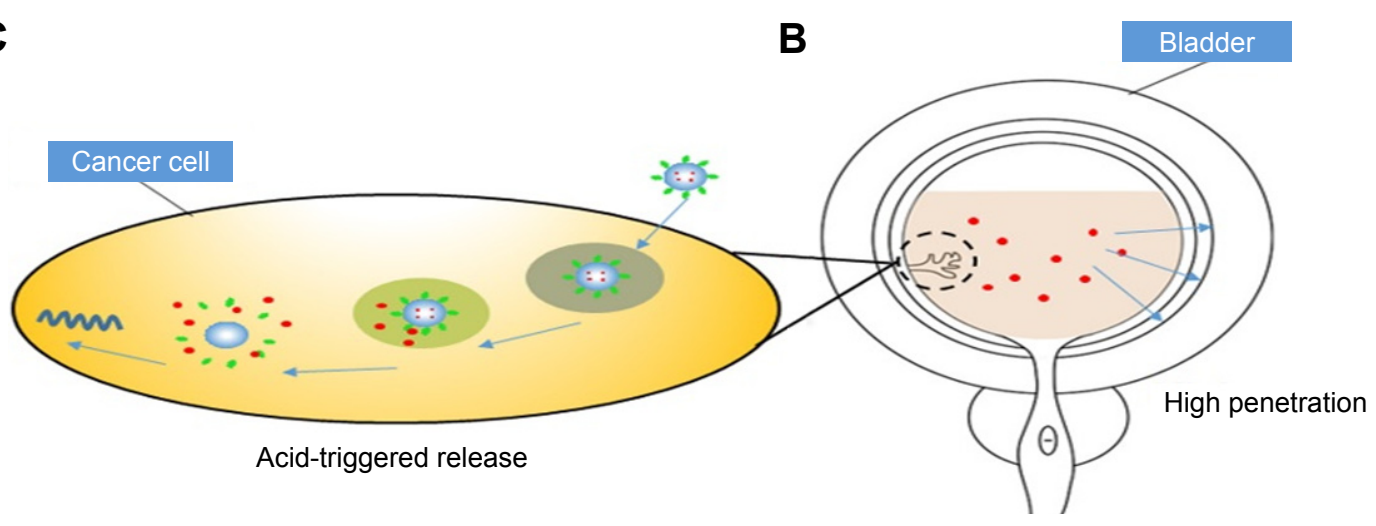

Figure I Schematic representation of the synthesis and working principle of high-penetrating, target-releasing PEG-PAMAM-DOX. Note: (A) Synthesis of PEG-PAMAM-DOX; (B) high penetration of PEG-PAMAM-DOX; (C) target-releasing of PEG-PMAMA-DOX Abbreviations: DOX, doxorubicin; PAMAM, polyamidoamine; PEG, polyethylene glycol.

administration and chemical approaches including instillation of chemical penetration enhancers such as dimethyl sulfoxide have been used to improve the penetration rate. ${ }^{5}$ Recently, many biomolecules such as chitosan, ${ }^{6-8}$ gelatin, ${ }^{9,10}$ hyperbranched polyglycerols, ${ }^{11,12}$ liposomes,${ }^{13}$ and poly (lactic-co-glycolic acid) (PLGA) $)^{2,14,15}$ have been developed to be nanocarriers to enhance the efficacy of intravesical instillation.

Dendrimers are highly branched three-dimensional macromolecules with highly controlled structures. Dendrimers have distinct advantages due to their highly ordered structure, narrow size distribution, availability of a large number of functional groups for attachment of drug molecules, and a high degree of control on the drug release properties, making them novel carriers for drug delivery. ${ }^{16-18}$ Polyamidoamine (PAMAM) dendrimers, which are prepared by a stepwise synthetic procedure, are the first complete dendrimer family. As a drug carrier, PAMAM has showed its great ability to penetrate cell speroids, ${ }^{19}$ oral mucosa, ${ }^{20}$ and the skin. ${ }^{21}$ Therefore, PAMAM is considered to be an applicable carrier for intravesical instillation, although it has not yet been explored. ${ }^{5}$

This study was therefore designed to develop a drug delivery system on the basis of PAMAM and polyethylene glycol (PEG) for the enhancement of intravesical instillation. Doxorubicin (DOX), a widely used chemotherapy drug for bladder cancer, was loaded into the core of PEG-PAMAM to form DOX-loaded nanoparticles (PEG-PAMAM-DOX). We hypothesized that PEG-PAMAM-DOX could penetrate BPB with high efficacy and release DOX from the nanoparticle in the acidic environment of cancer cells (Figure 1). To the best of our knowledge, it is the first study to investigate the enhancement of PEG-PAMAM-based nanocarriers for intravesical instillation.

\section{Materials and methods Materials}

PAMAM G5 and the components for preparation of artificial urine (urea, creatinine, $\mathrm{Na}_{3} \mathrm{C}_{6} \mathrm{H}_{5} \mathrm{O}_{7}, \mathrm{NaCl}, \mathrm{NH}_{4} \mathrm{Cl}, \mathrm{CaCl}_{2}$, $\mathrm{MgSO}_{4}, \mathrm{NaHCO}_{3}, \mathrm{Na}_{2} \mathrm{SO}_{4}, \mathrm{NaH}_{2} \mathrm{PO}_{4}$, and $\left.\mathrm{Na}_{2} \mathrm{HPO}_{4}\right)^{22}$ were purchased from Sigma-Aldrich (St Louis, MO, USA). MalPEG5K-NHS (PEG) was purchased from Seebio, Ltd (Shanghai, China). Doxorubicin ( $98 \%$, chemical grade) was purchased from Meilun Biology Technology Company, Ltd. (Dalian, Liaoning, China). The cell counting kit-8 (CCK-8) was supplied by Dojindo Laboratories (Kumamoto, Japan). Human bladder carcinoma cells MB-49 and human urothelial cells sv-huc-1 were obtained from the Model Animal Research Center of Nanjing University, China. All cell experiments were approved by the ethics committee of Nanjing University. Healthy, adult female ICR mice (around $20 \mathrm{~g}$ body weight) were obtained from the Experimental Animal Center (Yangzhou University, Yangzhou, China). Female C57BL/6 mice (around 20 g body weight) were purchased from the Model Animal Research Center of Nanjing University. All animal experiments were approved by the Institutional Animal Care and Use Committee of Nanjing University and followed the protocols approved by the Nanjing University Laboratory Animal Center. 


\section{Preparation of DOX-loaded PEG- PAMAM nanoparticles}

DOX-loaded PEG-PAMAM nanoparticles (PEG-PAMAMDOX) were prepared via a physical method as described previously. ${ }^{23}$ Briefly, PAMAM was conjugated with MalPEG5K-NHS at a 1:30 $\mathrm{M}$ ratio of PAMAM to PEG in a phosphate-buffered saline (PBS, $\mathrm{pH}=7.4$ ) for $30 \mathrm{~min}$ at room temperature. The resulting solution was then purified by Sephadex G-50 column to remove the unreacted PEG. After being freeze-dried for 2 days, the PEG-PAMAM complexes were obtained. To prepare PEG-PAMAM-DOX, PEG-PAMAM complexes $(10 \mathrm{mg})$ were dissolved in $2 \mathrm{~mL}$ water. Doxorubicin hydrochloride with 10 molar equivalents of dendrimers was dissolved in $400 \mu \mathrm{L}$ methanol and was neutralized with $5 \mu \mathrm{L}$ triethylamine to generate DOX. Then, the DOX solution was mixed with the dendrimer aqueous solution. The mixture solution was vigorously stirred overnight to allow the evaporation of the methanol solvent. Then, the mixture solution was centrifuged (7,000 rpm for $10 \mathrm{~min})$ to remove the free DOX.

\section{Characterization of PEG-PAMAM-DOX}

The particle size and zeta potential of PEG-PAMAM-DOX were determined by a DLS analyzer (90 Plus, Brookhaven Instruments Corporation, Holtsville, New York, USA). The temperature was kept at $37^{\circ} \mathrm{C}$ during the measuring process. All results were the mean of three test runs. The morphology of PEG-PAMAM-DOX was analyzed by transmission electron microscopy (TEM, JEM-2100, Hitachi H-7650, Japan).

The stability of PEG-PAMAM-DOX was tested by smearing the size change. The mean diameter of PEG-PAMAMDOX was investigated in PBS $(\mathrm{pH}=7.4)$ or artificial urine, respectively, at $0,1,2,4,8,12$, and $24 \mathrm{~h}$ to evaluate the stability of PEG-PAMAM-DOX.

The entrapment efficiency (EE) in PEG-PAMAM-DOX complexes was determined by UV-spectrophotometer (XXX). Lyophilized PEG-PAMAM-DOX complexes were dissolved in distilled water and the DOX concentration was quantified by UV absorbance (UV-2450, Shimadzu, Japan) at $497 \mathrm{~nm}$. EE was calculated as the ratio between the weight of DOX in conjugates and the weight of DOX fed initially.

\section{Cell viability assays}

Cell viability was determined by cell-counting kit-8 (CCK-8) assay (Jiancheng Biotechnology, Nanjing, China) to evaluate the potential cell toxicity of PAMAM. Briefly, MB-49 cells or sv-huc-1 cells were plated in 96-well flat-bottomed plates with a concentration of 5,000 cells per well and were allowed to be cultured with RPMI-1640 medium (Life Technology, Shanghai, China) supplemented with $10 \%$ fetal bovine serum (Life Technology) at $37^{\circ} \mathrm{C}$ under $5 \%$ $\mathrm{CO}_{2}$ for $24 \mathrm{~h}$ prior to incubation with PAMAM or PEGPAMAM at different concentrations of PAMAM from 0 to $800 \mu \mathrm{g} / \mathrm{mL}$. Forty-eight hours later, a culture medium containing PAMAM or PEG-PAMAM was changed with a fresh RPMI-1640 medium, and a reagent for determination was added to each well for an additional $2 \mathrm{~h}$. The cell viability was determined by measuring the absorption at $450 \mathrm{~nm}$ using a microplate reader (Molecular Devices M3, Beijing, China). Cells incubated without PAMAM or PEGPAMAM were set as control.

\section{Release study in vitro}

The release study of PEG-PAMAM-DOX was performed in artificial urine $(\mathrm{pH}=6.5)$ and in PBS with different $\mathrm{pH}$ conditions ( $\mathrm{pH}=7.4$ and $\mathrm{pH}=5.0$, respectively). To identify the release of DOX from PEG-PAMAM-DOX complexes in artificial urine, $0.5 \mathrm{~mL}$ of PEG-PAMAM-DOX solution was placed in a dialysis bag (molecular weight cutoff, $8 \mathrm{kDa}$ ). Dialysis bags were incubated in $20 \mathrm{~mL}$ of artificial urine as the release medium at $37^{\circ} \mathrm{C}$ with gentle shaking. At scheduled time points, $2.0 \mathrm{~mL}$ of release medium was withdrawn and replenished with an equal volume of fresh medium. The released DOX was quantified by UV spectrophotometry at $480 \mathrm{~nm}$. Free DOX was set as the control group. Similarly, the release of DOX from PEG-PAMAMDOX complexes was also identified in PBS with different $\mathrm{pH}$. Dialysis bags were incubated in $20 \mathrm{~mL}$ of PBS as the release medium.

\section{Penetration examination in vivo}

The capacity of PEG-PAMAM-DOX to enhance the permeability of instilled drugs was determined in vivo. Healthy, adult female ICR mice were divided into three groups, which were intravesically instilled with $0.5 \mathrm{~mL}$ of normal saline, free DOX $(200 \mu \mathrm{g} / \mathrm{mL})$, or PEG-PAMAM-DOX containing the same concentration of DOX, respectively. Intravesical administration was performed using an epidural anesthetic catheter inserted into the bladder through the urethra and tied in place by a ligature around the urethral orifice. Two hours later, all mice were euthanized and urinary bladders were harvested. The bladder was then washed with normal saline, followed by frozen section. The fluorescence of DOX in the bladder section was observed using a Zeiss MeBio fluorescence microscope (Nikon H600L, Japan). 


\section{Establishment of mice orthotopic bladder cancer models}

Female C57BL/6 mice were used according to protocols approved by the Nanjing University Laboratory Animal Center. Briefly, $7 \times 10^{5}$ MB- 49 cells in $0.1 \mathrm{~mL}$ PBS were injected into the bladder wall using $1 \mathrm{~mL}$ syringes after exposure of the bladder according to the previously described protocol. ${ }^{24}$

\section{In vivo therapeutic effect of PEG-PAMAM- DOX on inhibiting tumor growth}

Female C57BL/6 mice with orthotopic bladder cancer were randomly divided into three groups, which received intravesically an instillation of $100 \mu \mathrm{L}$ of normal saline, free DOX $(465 \mu \mathrm{g} / \mathrm{mL})$, or PEG-PAMAM-DOX containing the same concentration of DOX, respectively, at days 1, 5, 9, and 13 after tumor inoculation. The body weight of each mouse was recorded every day. All mice were euthanized and urinary bladders were harvested at day 18 . The tumor size was calculated as $V=d^{2} \times D / 2$, where $D$ and $d$ are the longest and shortest diameter of the tumor, respectively. After evaluation of tumor size, all bladder samples were fixed in formalin, processed routinely into paraffin, and sectioned for histological analysis. In addition, blood serum was collected for biochemistry assay of liver and renal function.

\section{Liver and renal function assessment and histology}

Alanine aminotransferase (ALT), aspartate aminotransferase (AST), blood urea nitrogen (BUN), and serum creatinine ( $\mathrm{Cr}$ ) were assessed using commercially available kits to reflect liver and kidney function. Bladder tissue sections $(5 \mu \mathrm{m})$ were stained with hematoxylin and eosin (HE) using a commercial available kit (Jiancheng Biotechnology, Nanjing, China) according to the protocol provided by the manufacturer.

\section{Statistical analysis}

Data were analyzed using Prism 4 (GraphPad Software, San Diego, CA, USA) and expressed as mean \pm SD. Multiple groups were compared using one-way analysis of variance followed by the Tukey-Kramer test for post hoc comparisons. Statistical significance was set at $P<0.05$.

\section{Results}

\section{Synthesis and characterization of PEG-} PAMAM-DOX

PEG-PAMAM-DOX was synthesized by the method previously reported ${ }^{23}$ In the present study, DOX was loaded into the relatively hydrophobic interior of the dendrimers by a physical mix. The EE of PEG-PAMAM-DOX was $85.2 \% \pm 1.5 \%$. The PEG-PAMAM-DOX solution is shown in Figure 2A, which is a light red transparent liquid. PEG-PAMAM-DOX are dispersed spherical morphology (Figure 2B) with an average diameter of $13 \mathrm{~nm}$ (Figure 2C). The polarity of PEG-PAMAM-DOX is weakly positive with the zeta potential of $+2.78 \mathrm{mV}$ (Figure 2D). The stability of PEG-PAMAM-DOX was investigated in artificial urine and PBS $(\mathrm{pH}=7.4)$ at $37^{\circ} \mathrm{C}$. The results revealed that the size of PEG-PAMAM-DOX remains consistent in both kinds of solution (Figure 2E), suggesting the stability of PEG-PAMAM-DOX.

\section{In vitro toxicity evaluation of PAMAM}

The potential toxicity of PAMAM to MB-49 cells or sv-huc-1 cells was measured to investigate the biocompatibility of PAMAM. As shown in Figure 3, PAMAM showed a significant concentration-depended toxicity to MB-49 cells and sv-huc-1 cells. After surface modification with PEG, PEGPAMAM did not show significant toxicity to either MB-49 cells or sv-huc-1 cells even at the highest concentration of PAMAM, indicating that surface modification with PEG remarkably improves the biocompatibility of PAMAM.

\section{DOX releases from PEG-PAMAM-DOX} in acidic solution in vitro

The release behavior of DOX from PEG-PAMAM-DOX complexes was first investigated in artificial urine. As shown in Figure 4A, the free DOX was quickly released in artificial urine. About $75 \%$ of DOX was released within $3 \mathrm{~h}$. In contrast, the cumulative release of DOX from the PEGPAMAM-DOX complexes showed that only a small amount of DOX was released. Only $23 \%$ of DOX was released at $3 \mathrm{~h}$, suggesting the role of PEG-PAMAM as an ideal drug carrier. Similarly, the release behavior of DOX form PEGPAMAM-DOX was investigated in PBS with different $\mathrm{pH}$ conditions. As shown in Figure 4B, only 37\% of DOX was released from PEG-PAMAM at $\mathrm{pH} 7.4$ after $24 \mathrm{~h}$. However, when $\mathrm{pH}$ decreased to 5.0 , about $60 \%$ DOX was released, indicating that acidic microenvironment could trigger the release of DOX from PEG-PAMAM.

\section{PEG-PAMAM penetrates bladder urothelium effectively}

The permeability of PEG-PAMAM-DOX was evaluated in mice bladder. Since DOX can be directly observed under fluorescent miscopy, the fluorescent intensity and penetration depth of DOX within the bladder wall were used to 
A

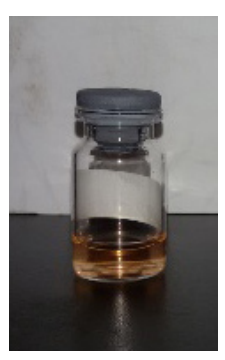

B

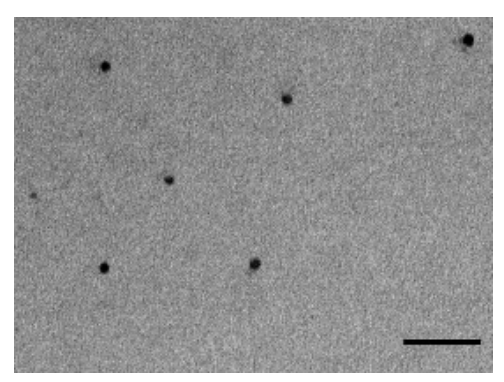

C

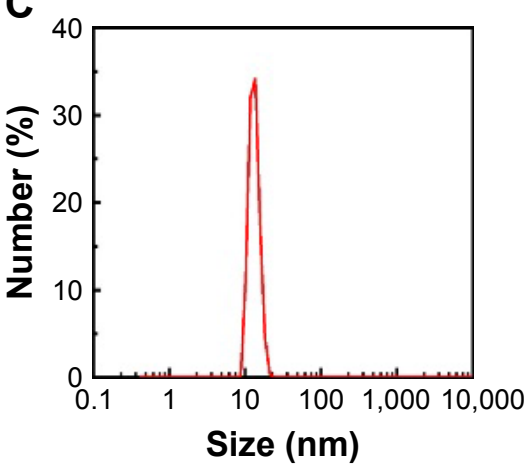

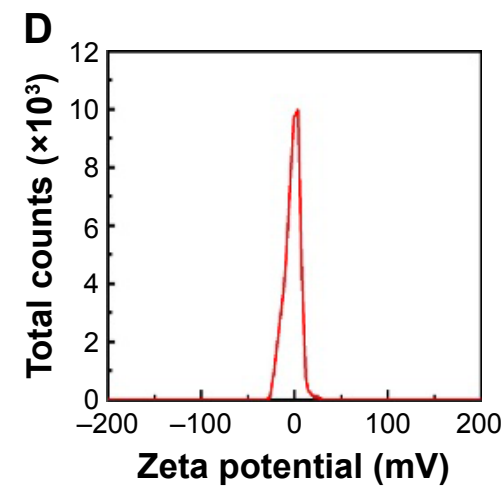

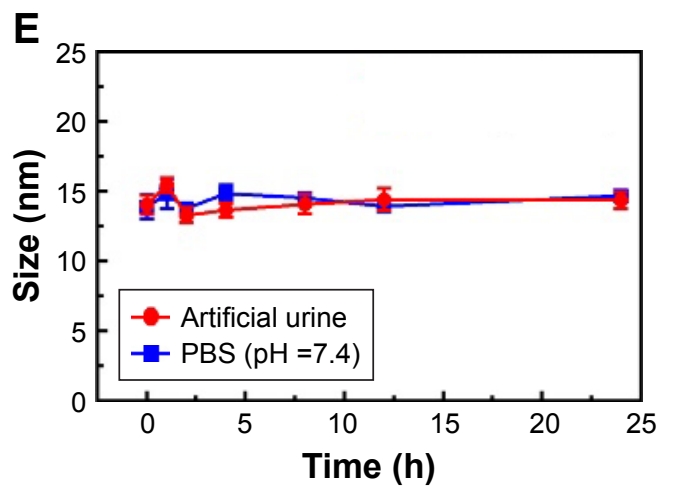

Figure 2 Characterization of PEG-PAMAM-DOX.

Notes: (A) General appearance of PEG-PAMAM-DOX solution; (B) Representative TEM image of PEG-PAMAM-DOX. Scale bar indicates $100 \mathrm{~nm}$; (C) Size distribution of PEG-PAMAM-DOX; (D) Zeta potential of PEG-PAMAM-DOX; (E) Change of particle size in a different solvent.

Abbreviations: DOX, doxorubicin; PAMAM, polyamidoamine; PBS, phosphate buffer saline; PEG, polyethylene glycol.

evaluate the penetrating property of PEG-PAMAM-DOX. As shown in Figure 5A, almost no red fluorescence was observed in the bladder wall instilled with normal saline. Very limited fluorescence was observed in the bladder wall instilled with free DOX. In contrast, significantly increased fluorescent intensity of DOX could be identified in the bladder wall instilled with PEG-PAMAM-DOX.
Furthermore, the semiquantitative results show a higher penetrating depth of DOX $(480 \pm 83 \mu \mathrm{m})$ and a greater fluorescent intensity of DOX $(4,050 \pm 2,810)$ in the bladder wall instilled with PEG-PAMAM-DOX compared with those $(860 \pm 96 \mu \mathrm{m}$ and $39,368 \pm 7,271$, respectively) in mice instilled with free DOX (Figure 5B and C). Taking together, PEG-PAMAM, as drug carrier, could penetrate
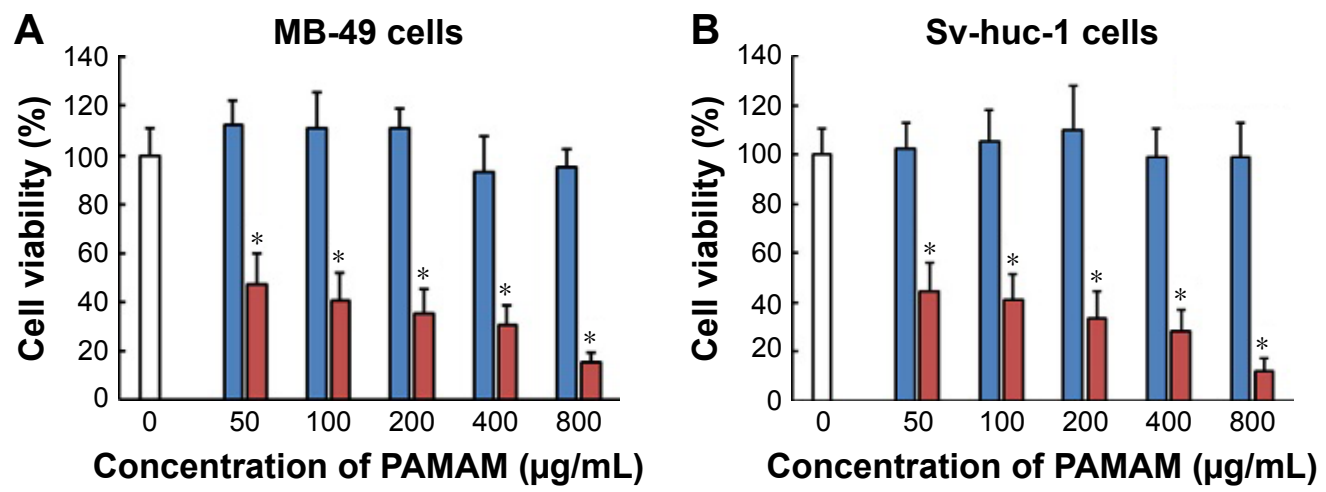

口 PEG-PAMAM $\square$ PAMAM

Figure 3 Toxicity of PAMAM to different cells.

Notes: Toxicity of PAMAM and PEG-PAMAM to MB-49 cells $(\mathbf{A})$ and sv-huc-I cells $(\mathbf{B})$ were evaluated by testing cell viability ( $\mathrm{n}=6$ ). Cells incubated without PAMAM or PEG-PAMAM were set as control. $* P<0.01$ compared with the control group.

Abbreviations: PAMAM, polyamidoamine; PEG, polyethylene glycol. 

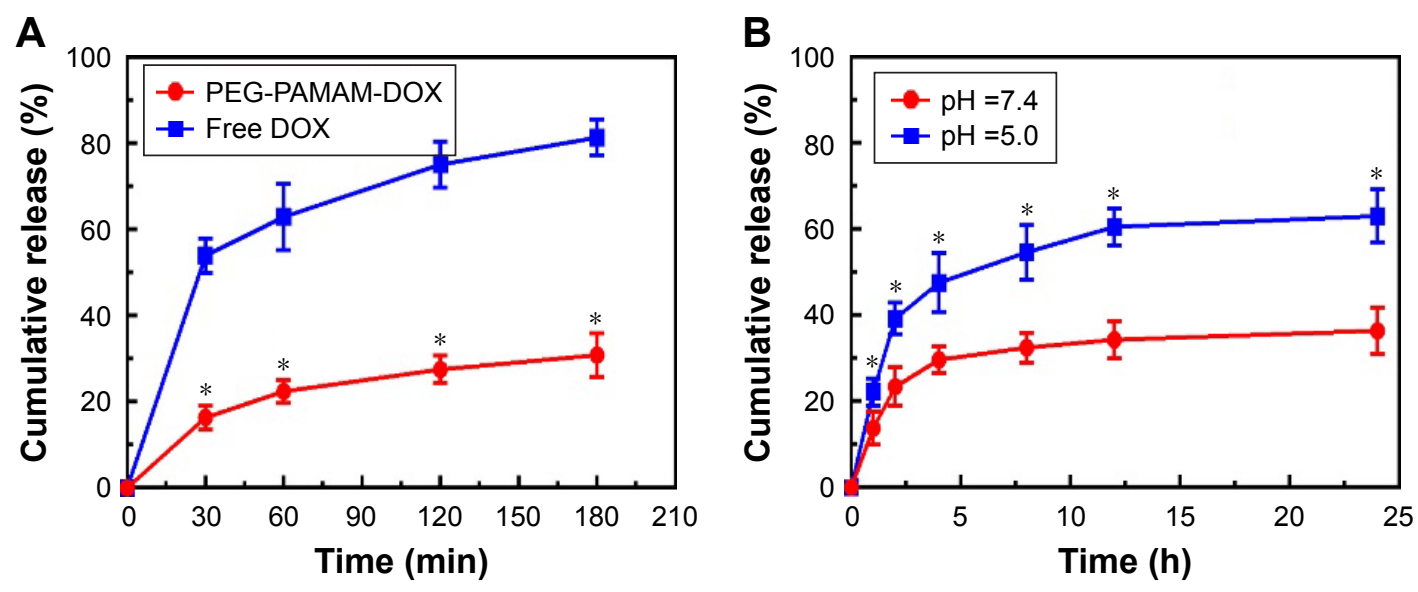

Figure 4 Release property of DOX from PEG-PAMAM-DOX in different conditions.

Notes: (A) Release behavior of DOX from PEG-PAMAM-DOX in artificial urine $(n=3)$. Free DOX was set as the control. (B) Release behavior of DOX from PEG-PAMAMDOX in different $\mathrm{pH}$ conditions $(\mathrm{pH}=7.4$ and $\mathrm{pH}=5.0)(\mathrm{n}=3) . * \mathrm{p}<0.05$.

Abbreviations: DOX, doxorubicin; PAMAM, polyamidoamine; PEG, polyethylene glycol.

bladder urothelium effectively and increased the amount of DOX within the bladder wall.

\section{PEG-PAMAM-DOX improves the efficacy of intravesical instillation}

The antitumor effect of PEG-PAMAM-DOX was evaluated using an orthotopic bladder cancer model in mice. The size of the tumor at the end of the experiments was measured and used to evaluate the antitumor effect. As shown in Figure 6, orthotopic bladder tumors grow rapidly in the control group without any treatment $\left(252 \pm 75 \mathrm{~mm}^{3}\right)$. The size of the tumor in mice instilled with free DOX was significantly smaller than those without any treatment, confirming the antitumor effects of DOX as a regular chemotherapeutic drug. Furthermore, a statistically significant attenuation of tumor volume was observed in mice instilled with the PEG-PAMAM-DOX $\left(75 \pm 32 \mathrm{~mm}^{3}\right)$ compared with those treated with free DOX $\left(15 \pm 5 \mathrm{~mm}^{3}\right)$, indicating that PEG-PAMAM-DOX improves the efficacy of DOX for intravesical instillation.

\section{PEG-PAMAM-DOX shows limited side effect}

To investigate the potential systematic toxicity of PEGPAMAM, the body weight of mice in each group was recorded and the function of liver and kidney assessed. As shown in Figure 7A, no significant difference was observed in body weight. ALT and AST were used to evaluate liver function, while $\mathrm{Cr}$ and BUN were used to evaluate renal function. As shown in Figure 7B, the level of ALT in each group was $45.32 \pm 4.79,48.18 \pm 3.21$, and $46.21 \pm 4.16 \mathrm{U} / \mathrm{L}$, respectively, while the level of AST in each group was $82.13 \pm 12.1$, $84.22 \pm 8.6$, and $83.12 \pm 8.9 \mathrm{U} / \mathrm{L}$, respectively. There was no significant difference between each group. The level of $\mathrm{Cr}$ in each group was $4.02 \pm 0.24,3.98 \pm 0.58$, and $3.87 \pm 0.32 \mu \mathrm{mol} / \mathrm{L}$
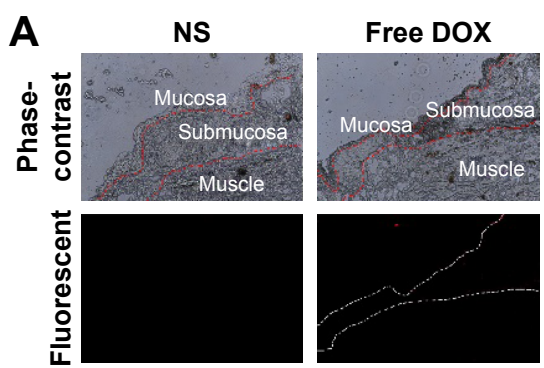

PEG-PAMAM-

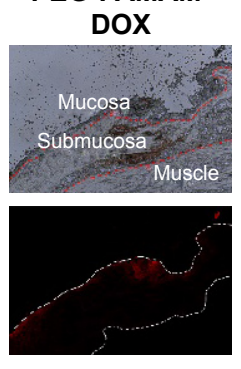

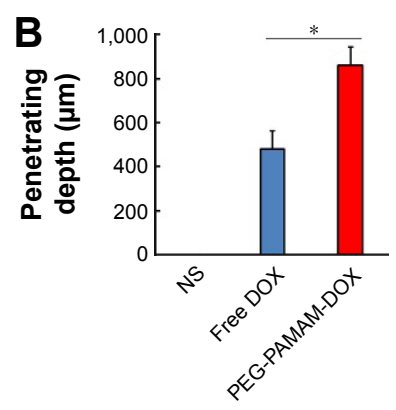

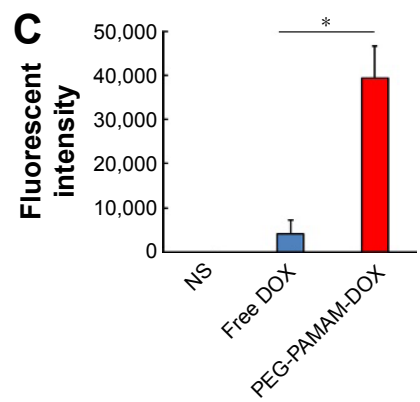

Figure 5 Penetrating property of PEG-PAMAM-DOX.

Notes: (A) Representative phase-contrast and fluorescent images of the bladder wall in each experimental group (200x). In the phase-contrast images, red lines distinguish the mucosa, submucosa, and muscle of the bladder wall. In the fluorescent images, white lines distinguish the penetrating depth of PEG-PAMAM-DOX. $(\mathbf{B}, \mathbf{C})$ Quantitative results of the penetrating depth of PEG-PAMAM-DOX $(\mathbf{B})$ and fluorescent intensity of penetrating DOX $(\mathbf{C})$ in the bladder wall $(\mathrm{n}=6)$. $* P<0.0 \mathrm{I}$ compared with NS group.

Abbreviations: DOX, doxorubicin; NS, normal saline; PAMAM, polyamidoamine; PEG, polyethylene glycol. 


\section{A}
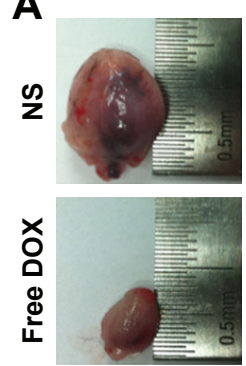

岂
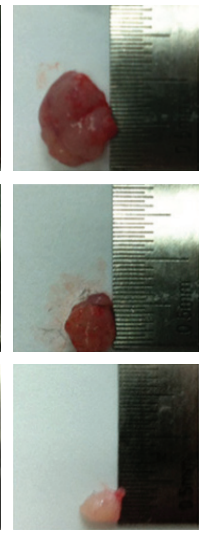
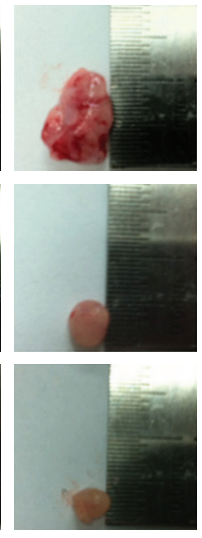
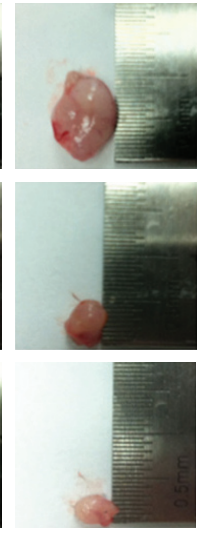

B

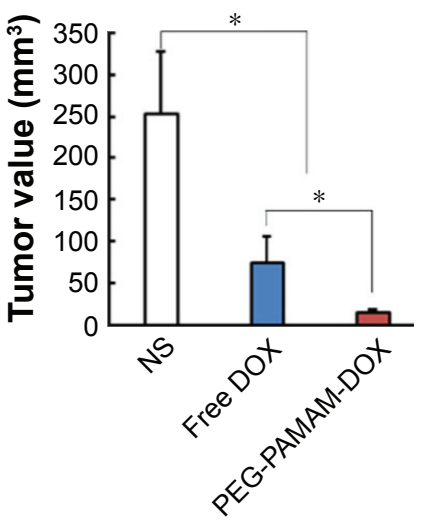

Figure 6 Antitumor effects of PEG-PAMAM-DOX for orthotopic bladder cancer.

Notes: (A) Images of orthotopic bladder tumor in each experimental group; (B) Results of tumor volume in different groups of mice $(n=4)$. $* p<0.05$.

Abbreviations: DOX, doxorubicin; NS, normal saline; PAMAM, polyamidoamine; PEG, polyethylene glycol.

A

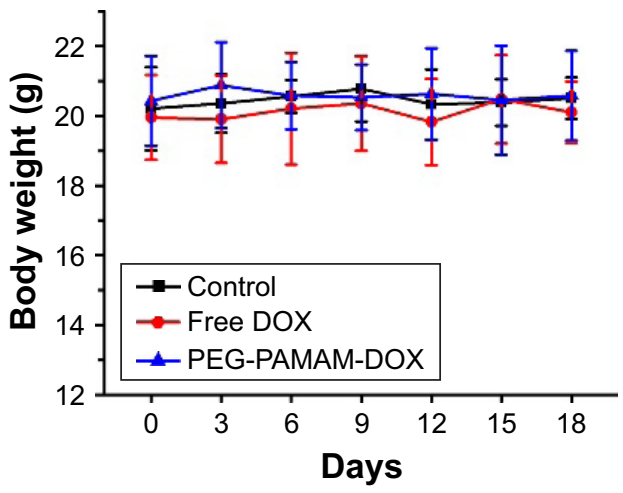

B
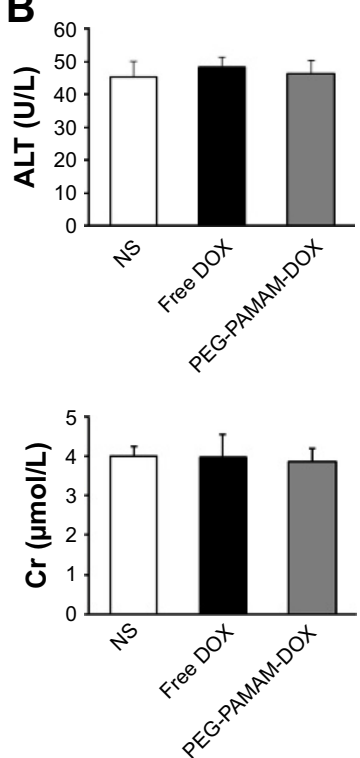

C

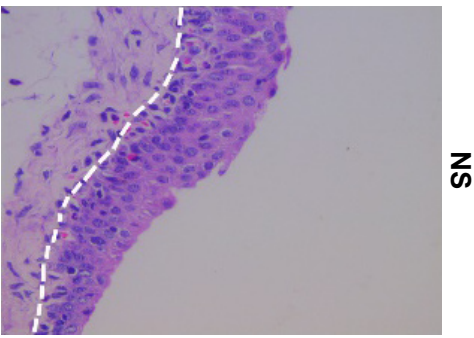

z
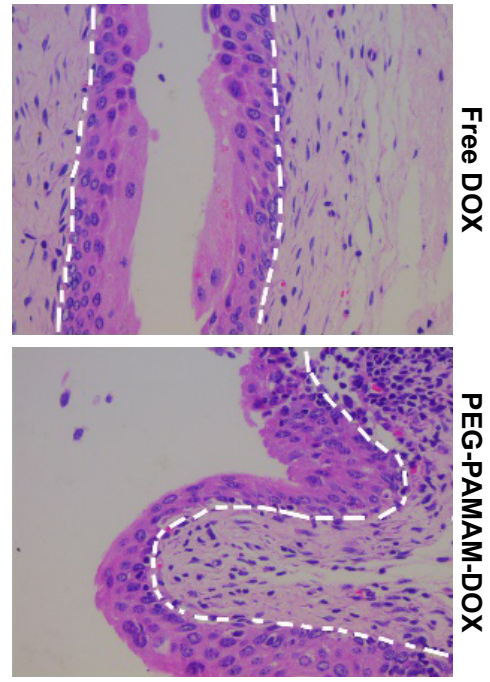

Figure 7 Potential toxicity of PAMAM to mice.

Notes: (A) Body weight of mice in each experimental group $(n=4)$. (B) Assessment of alanine aminotransferase (ALT), aspartate aminotransferase (AST), blood urea nitrogen $(B U N)$, serum creatinine $(\mathrm{Cr})$ in each experimental group. (C) Representative images of HE staining of the bladder wall in each experimental group (200x). The white line distinguishes the urothelium in each group.

Abbreviations: HE, hematoxylin and eosin; NS, normal saline; PAMAM, polyamidoamine. 
respectively, while the level of BUN in each group was $7.82 \pm 1.02,8.02 \pm 0.98$, and $8.18 \pm 0.882 \mathrm{mmol} / \mathrm{L}$, respectively. Similarly, there was no significant difference between each group. Furthermore, to investigate the potential toxicity of PEG-PAMAM on bladder urothelium, HE staining was used to detect the microstructure of the bladder wall in each experimental group. As shown in Figure 7C, the microstructure of urothelium treated with saline only is complete with dense arrangement of urothelial cells. In the mice treated with intravesical instillation of free DOX, cell density seemed to be reduced in urothelium, although the depth of the urothelium did not change, suggesting the toxicity of DOX to urothelium. In the mice treated with PEG-PAMAM-DOX, no severe injury to urothelium was observed.

\section{Discussion}

In the present study, we first applied PEG-PAMAM, a dendrimer, to develop a nanocarrier to deliver DOX to enhance the efficacy of intravesical instillation for the treatment of bladder cancer in a mice-orthotopic cancer model. We selected DOX as the loaded drug because DOX is a commonly used chemotherapeutic drug for intravesical instillation. ${ }^{25}$ Furthermore, DOX can be observed under the fluorescent microscope and makes it an ideal tracer in vivo. Our data indicated that PEG-PAMAM could penetrate the bladder urothelium and release DOX in cancer cells effectively, and therefore improve the antitumor effects of DOX.

Intravesical instillation of antitumor agents following TURBT has been considered as the gold standard strategy for the treatment of superficial bladder cancer. However, low permeability of urothelial layer allows very limited penetration of the drug into the bladder wall, resulting in low efficacy of intravesical instillation. ${ }^{26}$ Accordingly, the development of an effective drug delivery system to enhance the penetrating efficacy represents an important field of research. ${ }^{5}$ One of the commonly applied material is positively charged chitosan, which could bind to the negatively charged mucous membrane and affects the tight junctions of the umbrella cells, therefore enhancing permeability through the urothelial lining. ${ }^{27-29}$ However, the toxicity of chitosan to normal cell due to its highly positive charge is a big concern for further clinical application. ${ }^{30}$ Therefore, we evaluated the biocompatibility of PAMAM. As shown in the Results section, PAMAM, without any surface modification, showed significant toxicity to both normal urothelial cells and bladder cancers (Figure 3), which is consistent with the previously reported results. ${ }^{31,32}$ Previous study has demonstrated that PAMAM was nonimmunogenic had and low mammalian toxicity when its surface contains anionic or neutral groups. ${ }^{17}$ In the present study, PAMAM did not show significant toxicity to either normal urothelial cells or bladder cancer cells in vitro (Figure 3 ) after modification with PEG. Histological data from the bladder tissue did not show additional injury to normal urothelial cells in vivo (Figure 7), further confirming the biosafety of PEG-PAMAM.

Dendrimers were previously reported to be with high ability to penetrate intestinal mucosa, ${ }^{33}$ oral mucosa, ${ }^{20}$ and the skin. ${ }^{21}$ In the present study, we found that PEG-PAMAM could help DOX penetrate much deeper into bladder mucosa (Figure 5). The actual mechanism of how PEG-PAMAM could enhance urothelial permeability is not yet fully understood. It has been demonstrated that particle size has a substantial impact on the pharmacokinetics and biodistribution of nanoparticles. Some studies have pointed out that nanoparticles $<50 \mathrm{~nm}$ may penetrate into deeper tissue ${ }^{34,35}$ because larger particles may have poor delivery to less leaky sites in highly heterogeneous tumor tissues ${ }^{36}$ and narrow spacing between tumor cells. ${ }^{37,38}$ The diameter of a poly (amido amine) generation 5.0 (PAMAM G5) dendrimer used in our study is about $5.4 \mathrm{~nm}$. Even after surface modification with PEG and loading with DOX, the mean diameter of the complex is only about $13 \mathrm{~nm}$ (Figure 2), which is far smaller than most reported nanoparticles for intravesical instillation (usually $>100 \mathrm{~nm}$ ). Therefore, it is reasonable to speculate that PEG-PAMAM-DOX penetrates the urothelium effectively mainly due to its small particle size.

Target releasing is another distinguishing feature for an ideal drug delivery system. As described before, the core of PAMAM allows the encapsulation of hydrophobic anticancer drugs, with a high payload. ${ }^{23}$ Therefore, doxorubicin hydrochloride was first desalinated to generate DOX for encapsulation into PEG-PAMAM in the present study. Sluggish drug release has been demonstrated in PEGylated PAMAM dendrimers. ${ }^{39}$ We then investigated the release property of PEG-PAMAM-DOX in artificial urine. The results indicated that only $23 \%$ DOX was released from PEG-PAMAM-DOX in $2 \mathrm{~h}$, suggesting the ineffective and slow DOX release from PEG-PAMAM-DOX in artificial urine. We further investigated the release of DOX from PEG-PAMAM-DOX in a different $\mathrm{pH}$ condition. The results indicated that DOX could be released effectively when $\mathrm{pH}$ decreased to 5.0 (Figure 4), which is similar to the acidic microenvironment in cancer cells. This might be due to the structural change that PEGPAMAM undergoes, from a dense core to a dense shell at low $\mathrm{pH}$ condition ${ }^{40}$ so that the loaded DOX in the core of PEG-PAMAM could be released. Taking together, the release 
of DOX from PEG-PAMAM-DOX is sluggish, which is beneficial for intravesical instillation since a limited part of DOX is released to urine. Furthermore, PEG-PAMAMDOX could be considered a target-releasing drug delivery system triggered by cancer cells that are specific to an acidic microenvironment, making it an ideal drug delivery system for intravesical instillation. Considering the advantages of the PEG-PAMAM-based delivery system, some other therapeutic agents such as suicide genes, ${ }^{41}$ bacterial minicell, ${ }^{42}$ and protein-loaded liposome system ${ }^{43}$ could be encapsulated into the delivery system.

Our study has some limitations. First, intravesical instillation of chemotherapeutic agents are used for patients with nonmuscular-invasive bladder cancer following TURBT. In the present study, we could not define the clinical stage of the orthotopic bladder cancer. Furthermore, DOX or PEGPAMAM-DOX was administrated in the bladder without resection of the orthotopic cancer, which is different from clinical practice. Second, $\mathrm{pH}$-response release was observed only in the in vitro study. Our present study could not provide direct evidence of target releasing of PEG-PAMAM-DOX in tumor tissue.

\section{Conclusion}

In this study, we successfully developed a drug delivery system on the basis of DOX and PEG-PAMAM. PEG-PAMAM could help DOX to penetrate urothelium and release DOX in cancer cells that are specific to an acidic microenvironment, making it an ideal drug delivery system for intravisical instillation.

\section{Acknowledgments}

This study was supported by grants from the National Natural Science Foundation of China (81602232, 81572519, 81572519), Natural Science Foundation of Jiangsu Province (BK20150112, BK20150097), and Nanjing Health Distinguished Youth Fund (JQX16025).

\section{Disclosure}

The authors report no conflicts of interest in this work.

\section{References}

1. Siegel RL, Miller KD, Jemal A. Cancer statistics, 2015. CA Cancer J Clin. 2015;65(1):5-29.

2. Martin DT, Hoimes CJ, Kaimakliotis HZ, et al. Nanoparticles for urothelium penetration and delivery of the histone deacetylase inhibitor belinostat for treatment of bladder cancer. Nanomedicine. 2013;9(8): 1124-1134.

3. Clark PE, Agarwal N, Biagioli MC, et al; National Comprehensive Cancer Network (NCCN). Bladder cancer. J Natl Compr Canc Netw. 2013;11(4):446-475.
4. Babjuk M, Burger M, Zigeuner R, et al; European Association of Urology. EAU guidelines on non-muscle-invasive urothelial carcinoma of the bladder: update 2013. Eur Urol. 2013;64(4):639-653.

5. GuhaSarkar S, Banerjee R. Intravesical drug delivery: challenges, current status, opportunities and novel strategies. J Control Release. 2010;148(2):147-159.

6. Barthelmes J, Perera G, Hombach J, Dunnhaupt S, Bernkop-Schnurch A. Development of a mucoadhesive nanoparticulate drug delivery system for a targeted drug release in the bladder. Int J Pharm. 2011; 416(1):339-345.

7. Barthelmes J, Dunnhaupt S, Unterhofer S, Perera G, Schlocker W, Bernkop-Schnurch A. Thiolated particles as effective intravesical drug delivery systems for treatment of bladder-related diseases. Nanomedicine (Lond). 2013;8(1):65-75.

8. Erdogar N, Iskit AB, Eroglu H, Sargon MF, Mungan NA, Bilensoy E. Cationic core-shell nanoparticles for intravesical chemotherapy in tumor-induced rat model: safety and efficacy. Int J Pharm. 2014; 471(1-2):1-9.

9. Lu Z, Yeh TK, Tsai M, Au JL, Wientjes MG. Paclitaxel-loaded gelatin nanoparticles for intravesical bladder cancer therapy. Clin Cancer Res. 2004;10(22):7677-7684.

10. Lu Z, Yeh TK, Wang J, et al. Paclitaxel gelatin nanoparticles for intravesical bladder cancer therapy. J Urol. 2011;185(4):1478-1483.

11. Mugabe C, Matsui Y, So AI, et al. In vivo evaluation of mucoadhesive nanoparticulate docetaxel for intravesical treatment of non-muscleinvasive bladder cancer. Clin Cancer Res. 2011;17(9):2788-2798.

12. Mugabe C, Raven PA, Fazli L, et al. Tissue uptake of docetaxel loaded hydrophobically derivatized hyperbranched polyglycerols and their effects on the morphology of the bladder urothelium. Biomaterials. 2012;33(2):692-703.

13. Kang MR, Yang G, Place RF, et al. Intravesical delivery of small activating RNA formulated into lipid nanoparticles inhibits orthotopic bladder tumor growth. Cancer Res. 2012;72(19):5069-5079.

14. Neutsch L, Wirth EM, Spijker S, et al. Synergistic targeting/prodrug strategies for intravesical drug delivery-lectin-modified PLGA microparticles enhance cytotoxicity of stearoyl gemcitabine by contactdependent transfer. J Control Release. 2013;169(1-2):62-72.

15. Martin DT, Steinbach JM, Liu J, et al. Surface-modified nanoparticles enhance transurothelial penetration and delivery of survivin siRNA in treating bladder cancer. Mol Cancer Ther. 2014;13(1):71-81.

16. Svenson S. Dendrimers as versatile platform in drug delivery applications. Eur J Pharm Biopharm. 2009;71(3):445-462.

17. Esfand R, Tomalia DA. Poly(amidoamine) (PAMAM) dendrimers: from biomimicry to drug delivery and biomedical applications. Drug Discov Today. 2001;6(8):427-436.

18. Patri AK, Myc A, Beals J, Thomas TP, Bander NH, Baker JR Jr. Synthesis and in vitro testing of J591 antibody-dendrimer conjugates for targeted prostate cancer therapy. Bioconjug Chem. 2004;15(6): 1174-1181.

19. Yuan A, Yang B, Wu J, Hu Y, Ming X. Dendritic nanoconjugates of photosensitizer for targeted photodynamic therapy. Acta Biomater. 2015;21(2015):63-73.

20. Duncan R, Izzo L. Dendrimer biocompatibility and toxicity. Adv Drug Deliv Rev. 2005;57(15):2215-2237

21. Wang Z, Itoh Y, Hosaka Y, et al. Novel transdermal drug delivery system with polyhydroxyalkanoate and starburst polyamidoamine dendrimer. J Biosci Bioeng. 2003;95(5):541-543.

22. Chutipongtanate S, Thongboonkerd V. Systematic comparisons of artificial urine formulas for in vitro cellular study. Anal Biochem. 2010; 402(1):110-112.

23. Hu W, Cheng L, Cheng L, et al. Redox and $\mathrm{pH}$-responsive poly (amidoamine) dendrimer-poly (ethylene glycol) conjugates with disulfide linkages for efficient intracellular drug release. Colloids Surf B Biointerfaces. 2014;123:254-263.

24. Miller A, Burson H, Söling A, Roughan J. Welfare assessment following heterotopic or orthotopic inoculation of bladder cancer in C57BL/6 Mice. PLoS One. 2016;11(7):e0158390. 
25. See WA, Dreicer R, Forest PK, Riggs C. Continuous infusion, intravesical doxorubicin for the treatment of regionally advanced bladder cancer: a phase I-II trial. Am J Clin Oncol. 1997;20(4):331-337.

26. Au JL, Jang SH, Wientjes MG. Clinical aspects of drug delivery to tumors. J Control Release. 2002;78(1-3):81-95.

27. Kerec M, Bogataj M, Veranic P, Mrhar A. Permeability of pig urinary bladder wall: the effect of chitosan and the role of calcium. Eur J Pharm Sci. 2005;25(1):113-121.

28. Eroğlu M, Irmak S, Acar A, Denkbaş EB. Design and evaluation of a mucoadhesive therapeutic agent delivery system for postoperative chemotherapy in superficial bladder cancer. Int J Pharm. 2002;235(1-2): 51-59.

29. Burjak M, Bogataj M, Velnar M, Grabnar I, Mrhar A. The study of drug release from microspheres adhered on pig vesical mucosa. Int J Pharm. 2001;224(1-2):123-130.

30. Kos MK, Bogataj M, Veranic P, Mrhar A. Permeability of pig urinary bladder wall: time and concentration dependent effect of chitosan. Biol Pharm Bull. 2006;29(8):1685-1691.

31. Han L, Huang R, Liu S, Huang S, Jiang C. Peptide-conjugated PAMAM for targeted doxorubicin delivery to transferrin receptor overexpressed tumors. Mol Pharm. 2010;7(6):2156-2165.

32. Jones CF, Campbell RA, Franks Z, et al. Cationic PAMAM dendrimers disrupt key platelet functions. Mol Pharm. 2012;9(6):1599-1611.

33. Jevprasesphant R, Penny J, Attwood D, McKeown NB, D'Emanuele A. Engineering of dendrimer surfaces to enhance transepithelial transport and reduce cytotoxicity. Pharm Res. 2003;20(10):1543-1550.

34. Wong C, Stylianopoulos T, Cui J, et al. Multistage nanoparticle delivery system for deep penetration into tumor tissue. Proc Natl Acad Sci US A. 2011;108(6):2426-2431.
35. Kim HJ, Takemoto H, Yi Y, et al. Precise engineering of siRNA delivery vehicles to tumors using polyion complexes and gold nanoparticles. ACS Nano. 2014;8(9):8979-8991.

36. Ruenraroengsak P, Cook JM, Florence AT. Nanosystem drug targeting: Facing up to complex realities. J Control Release. 2010;141(3): 265-276.

37. Nagano S, Perentes JY, Jain RK, Boucher Y. Cancer cell death enhances the penetration and efficacy of oncolytic herpes simplex virus in tumors. Cancer Res. 2008;68(10):3795-3802.

38. Ishida $\mathrm{T}$, Kiwada $\mathrm{H}$. Alteration of tumor microenvironment for improved delivery and intratumor distribution of nanocarriers. Biol Pharm Bull. 2013;36(5):692-697.

39. Liu D, Hu H, Zhang J, Zhao X, Tang X, Chen D. Drug pH-sensitive release in vitro and targeting ability of polyamidoamine dendrimer complexes for tumor cells. Chem Pharm Bull (Tokyo). 2011;59(1):63-71.

40. Liu Y, Bryantsev VS, Diallo MS, Goddard WA 3rd. PAMAM dendrimers undergo $\mathrm{pH}$ responsive conformational changes without swelling. J Am Chem Soc. 2009;131(8):2798-2799.

41. Chen X, Scapa JE, Liu DX, Godbey WT. Cancer-specific promoters for expression-targeted gene therapy: ran, brms1 and mcm5. J Gene Med. 2016;18(7):89-101.

42. Tsuji S, Chen X, Hancock B, et al. Preclinical evaluation of VAX-IP, a novel bacterial minicell-based biopharmaceutical for nonmuscle invasive bladder cancer. Mol Ther Oncolytics. 2016;3:16004. doi:10.1038/ mto.2016.4.

43. Vila-Caballer M, Codolo G, Munari F, et al. A pH-sensitive stearoylPEG-poly(methacryloyl sulfadimethoxine)-decorated liposome system for protein delivery: an application for bladder cancer treatment. J Control Release. 2016;238:31-42.
International Journal of Nanomedicine

\section{Publish your work in this journal}

The International Journal of Nanomedicine is an international, peerreviewed journal focusing on the application of nanotechnology in diagnostics, therapeutics, and drug delivery systems throughout the biomedical field. This journal is indexed on PubMed Central, MedLine, CAS, SciSearch $\AA$, Current Contents ${ }^{\circledR} /$ Clinical Medicine,

\section{Dovepress}

Journal Citation Reports/Science Edition, EMBase, Scopus and the Elsevier Bibliographic databases. The manuscript management system is completely online and includes a very quick and fair peer-review system, which is all easy to use. Visit http://www.dovepress.com/ testimonials.php to read real quotes from published authors. 